\title{
The Key Indicator for the Control of Metal Particle Sizes on Supports from First Principles and Experimental Observation
}

\author{
Takuya Nakao, ${ }^{\dagger}$ Tomofumi Tada, ${ }^{*}$ Masaaki Kitano, ${ }^{*}$ Masato Sasase ${ }^{\dagger, t}$ Hideo Hosono ${ }^{*}, \ldots, \S$ \\ ${ }^{\dagger}$ Laboratory for Materials and Structures, Tokyo Institute of Technology, 4259 Nagatsuta, Midori-ku,
}

Yokohama 226-8503, Japan

${ }^{\ddagger}$ Materials Research Center for Element Strategy, Tokyo Institute of Technology, 4259 Nagatsuta,

Midori-ku, Yokohama 226-8501, Japan

${ }^{\S}$ ACCEL, Japan Science and Technology Agency, 4-1-8 Honcho, Kawaguchi, Saitama, 332-0012, Japan.

E-mail: hosono@msl.titech.ac.jp

Phone: +81-45-924-5009

Table of Contents

1. Calculated surface energies of the supports $\quad$ S2

2. Comparison between PDOS calculated using VASP and that using SIESTA S3

3. Calculated COHP of Ru-anion in the supports S5

4. Ru migration pathways $\quad$ S6

5. Transmission electron microscope (TEM) and scanning TEM (STEM) $\begin{array}{ll}\text { images of Ru loaded the supports } & \text { S7 }\end{array}$

$\begin{array}{ll}\text { 6. References } & \text { S8 }\end{array}$ 


\section{Calculated surface energies of the supports}

To make Ru-adsorbed support models, we first determined the most stable surface of support

materials among the low-index surfaces. Table S1 lists the calculated surface energies of $\mathrm{CaO}, \mathrm{Ca}_{3} \mathrm{~N}_{2}$, and $\mathrm{Ca}_{2} \mathrm{Si}$ obtained with DFT calculations. For $\mathrm{CaF}_{2}$, on the other hand, we used the surface energies

calculated by Puchin et al ${ }^{1}$, which are also listed in Table S1. The surface energy $\gamma_{i j k}$ is defined in this study as

$\gamma_{i j k}=\left(E_{\text {surf }}-N E_{\text {bulk }}\right) / 2 S_{i j k}$

where $E_{\text {surf }}$ is the total energy per unit cell of (ijk) surface (i.e., slab) model, $E_{\text {bulk }}$ is the total energy

of bulk per atom, $N$ stands for the number of atoms in the surface model, and $S_{i j k}$ is the surface area of (ijk) crystallographic plane.

First we explain the model construction of the (111), (110) and (100) surfaces of $\mathrm{CaF}_{2}$ reported by Puchin et al ${ }^{1}$. The surface unit of the (111) surface is composed of $\mathrm{F}^{-}-\mathrm{Ca}^{2+}-\mathrm{F}^{-}$three layers and thus the surface is the neutral (i.e., non-polarized) cleavage planes of the crystal. The layer of (110) surface contains one $\mathrm{Ca}^{2+}$ and two $\mathrm{F}^{-}$ions per layer, and thus the (110) surface also can be the neutral planes . On the other hand, since $\mathrm{CaF}_{2}$ crystal is composed of alternating $\mathrm{Ca}^{2+}$ and $\mathrm{F}^{-}$layers in the (100) directions, (100) surfaces are polar surfaces depending on the surface termination (i.e., $\mathrm{Ca} / \mathrm{F}$ termination leads to positively/negatively polarized surface). In a standard model construction for $\mathrm{CaF}_{2}(100)$ slab, both $\mathrm{Ca}$ and $\mathrm{F}$ terminations appear in the stoichiometric model, and thereby appropriate structural calculations and evaluations of surface energies are difficult because of the 
presence of artificial dipole-dipole interactions between the Ca- and F-terminated surfaces. To avoid this problem, Puchin et al. moved the half of the surface $\mathrm{F}^{-}$ions from the F-terminated surface to Ca-terminated surface so as to cancel the artificial dipole-dipole interactions between the two surfaces. Based on this model concept, they carried out the structural relaxation of $\mathrm{CaF}_{2}(100)$ surface and estimated the surface energy of the (100) surface. According to the calculated surface energies of $\mathrm{CaF}_{2}$ by Puchin et al ${ }^{1}$, the $\gamma_{100}\left(\mathrm{CaF}_{2}\right)$ is larger than the $\gamma_{110}\left(\mathrm{CaF}_{2}\right)$ and $\gamma_{111}\left(\mathrm{CaF}_{2}\right)$. In other words, the neutral planes of $\mathrm{CaF}_{2}$ (i.e., (111) and (110) planes) are more stable than the polarized plane of $\mathrm{CaF}_{2}$ (i.e., (100) plane). The (111) surface is consequently the most stable surface among the low-index surfaces.

The surface models of $\mathrm{CaO}$ and $\mathrm{Ca}_{3} \mathrm{~N}_{2}$ especially for polarized surfaces were constructed in the same manner by Puchin et al. We calculated the surface energies of (100) and (110) surfaces of $\mathrm{CaO}$, and (100) and (110) surfaces of $\mathrm{Ca}_{3} \mathrm{~N}_{2}$. The (100) and (110) surfaces of $\mathrm{CaO}$ and (110) surfaces of $\mathrm{Ca}_{3} \mathrm{~N}_{2}$ can be neutral planes, and (100) surfaces of $\mathrm{Ca}_{3} \mathrm{~N}_{2}$ are polar surfaces depending on the surface termination. From the calculated results, we found that the $\gamma_{100}(\mathrm{CaO})$ is smaller than $\gamma_{110}(\mathrm{CaO})$, and the $\gamma_{110}\left(\mathrm{Ca}_{3} \mathrm{~N}_{2}\right)$ is smaller than $\gamma_{100}\left(\mathrm{Ca}_{3} \mathrm{~N}_{2}\right)$.

Therefore, we used $\mathrm{CaF}_{2}$ (111), $\mathrm{CaO}(100)$ and $\mathrm{Ca}_{3} \mathrm{~N}_{2}$ (110) as $\mathrm{Ru}$ - loaded surfaces. We also found the neutral surfaces are clearly more stable than the originally polarized surfaces. Thus, we used (001) surface of $\mathrm{Ca}_{2} \mathrm{Si}$ as Ru-loaded surfaces since the other low-index planes of $\mathrm{Ca}_{2} \mathrm{Si}$ are the polarized planes. 
Table S1. Calculated Surface Energies of $\mathrm{Ca}_{3} \mathrm{~N}_{2}, \mathrm{CaO}$, and $\mathrm{CaF}_{2}$

\begin{tabular}{cccc}
\hline & Surface index & Vacuum width $[\AA]$ & Surface energy $\left[\mathrm{J} / \mathrm{m}^{2}\right]$ \\
\hline $\mathrm{Ca}_{3} \mathrm{~N}_{2}$ & $(100)$ & 15 & 1.383 \\
& $(110)$ & 15 & 0.989 \\
$\mathrm{CaO}$ & $(100)$ & 15 & 0.637 \\
& $(110)$ & 15 & 1.470 \\
$\mathrm{CaF}_{2}$ & $(100)$ & $13.80^{1}$ & $1.189^{1}$ \\
& $(110)$ & $11.70^{1}$ & $0.819^{1}$ \\
& $(111)$ & $11.20^{1}$ & $0.467^{1}$ \\
\hline
\end{tabular}

\section{Comparison between PDOS Calculated by Using VASP and SIESTA}

In this study, we calculated the adsorption and migration energies of a $\mathrm{Ru}$ atom on the $\mathrm{Ca}_{3} \mathrm{~N}_{2}, \mathrm{CaO}$,

$\mathrm{CaF}_{2}$ and $\mathrm{Ca}_{2} \mathrm{Si}$ by using VASP, and the $\mathrm{COHP}$ of $\mathrm{Ru}$-anion pairs in the $\mathrm{Ru}-\mathrm{Ca}_{3} \mathrm{~N}_{2}, \mathrm{CaO}, \mathrm{CaF}_{2}$ and

$\mathrm{Ca}_{2} \mathrm{Si}$ by using SIESTA to compare the strength of covalent bonds between $\mathrm{Ru}$ atom and the anions

in the four supports. Since the basis functions and the handling of core electrons are different in the two DFT programs, the electronic structures of materials obtained with VASP will not be identical to those obtained with SIESTA even when the crystal structures are the same in both methods. The details of the DOS profiles actually depend on the DFT programs. Therefore we have to carefully check the validity of chemical bond analysis with SIESTA based on model structures obtained with VASP.

In bond analysis, we focused our attention on the chemical bonds between a $\mathrm{Ru}$ atom and the surface anions of the four supports. When the DOS and partial DOS (PDOS) profiles of a Ru atom and the anions bonded with $\mathrm{Ru}$ of the four supports by using VASP show good correspondence with those with SIESTA, the present strategy (i.e., structures from VASP and bond analysis with SIESTA) 
can be an acceptable analysis.

Fig. S1(a-d) shows the PDOS of $\mathrm{N}$ and $\mathrm{F}$ bonded with $\mathrm{Ru}$ and a $\mathrm{Ru}$ atom on the $\mathrm{Ca}_{3} \mathrm{~N}_{2}$ and $\mathrm{CaF}_{2}$ calculated with VASP and SIESTA. The peak positions and the number of the peaks of $\mathrm{N}$ and $\mathrm{Ru}$ relating to the N-Ru chemical bond obtained with VASP (Peaks 1-6 in Fig. S1(a)) clearly correspond with those with SIESTA (Peaks 1-6 in Fig. S1(b)). We also found the same tendency of the PDOS of F and Ru (See Peaks 7-11 in Fig S1(c) and S1(d)). The same trend was also obtained about Ru-O and Si. Therefore, we confirmed the validity of the bond analysis with SIESTA. As a result, the trend of the adsorption and migration energies of Ru atom on the four supports calculated with VASP shows a clear correspondence with that of the chemical bond strength of Ru-anions pairs calculated with SIESTA. 
(a)

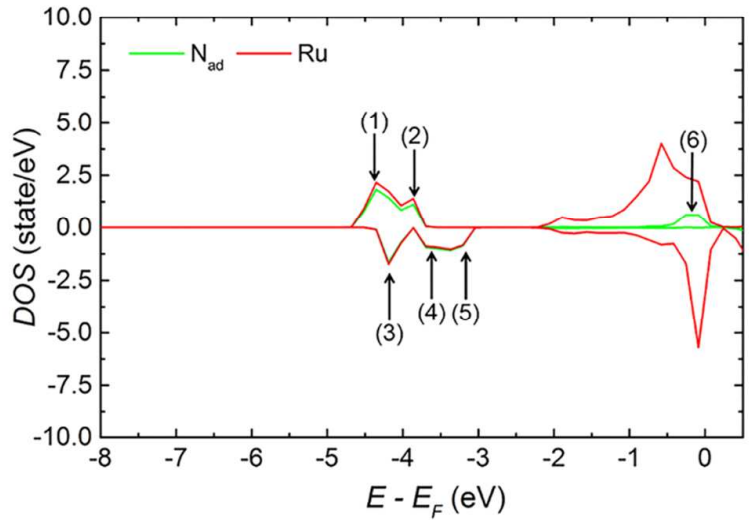

(c)

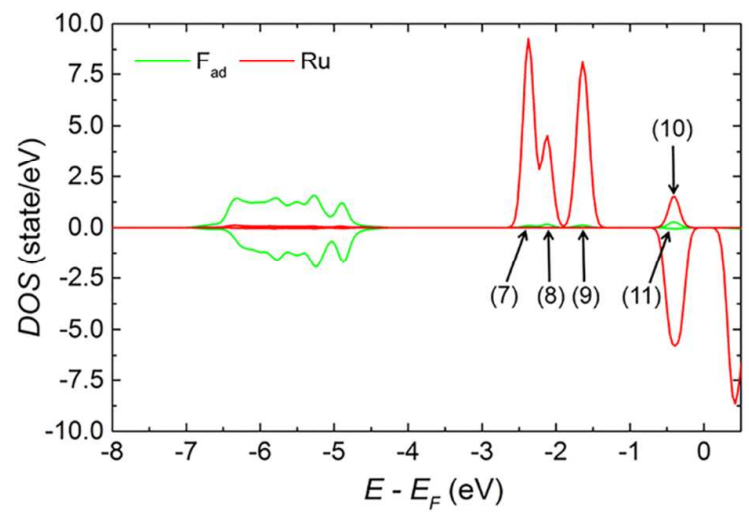

(b)

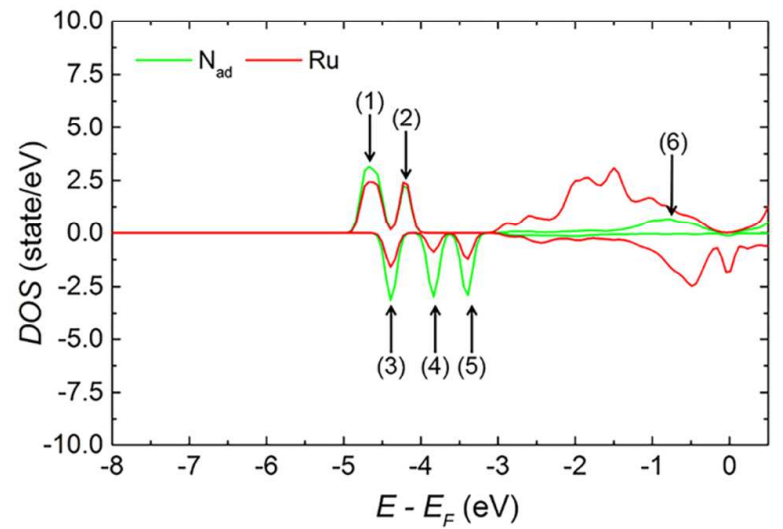

(d)

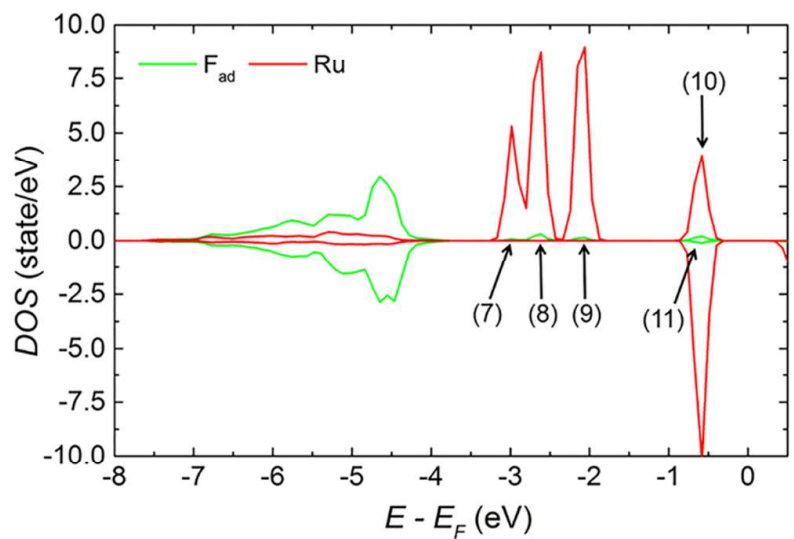

Figure S1. Calculated PDOS of the $\mathrm{N}$ and $\mathrm{Ru}$ atoms in $\mathrm{N}-\mathrm{Ru}$ bond calculated with (a) VASP and (b) SIESTA, and calculated PDOS of F and Ru in F-Ru bond with (c) VASP and (d) SIESTA. 


\section{Calculated COHP of Ru-anion in the supports}

To evaluate the bond strength of Ru-anion quantitatively, we calculated the COHP and ICOHP of

Ru-anion pairs in the four supports by using SIESTA. Figure S2 shows the calculated COHP of

Ru-anion in the $\mathrm{Ca}_{3} \mathrm{~N}_{2}, \mathrm{CaO}, \mathrm{CaF}_{2}$ and $\mathrm{Ca}_{2} \mathrm{Si}$. We obtained the ICOHP values by integrating COHP in Fig. S2 from a sufficiently low energy level to the Fermi level.

(a)

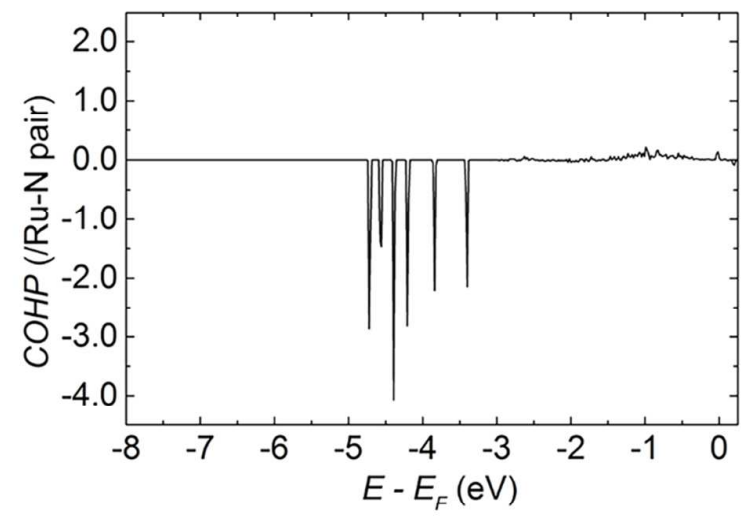

(c)

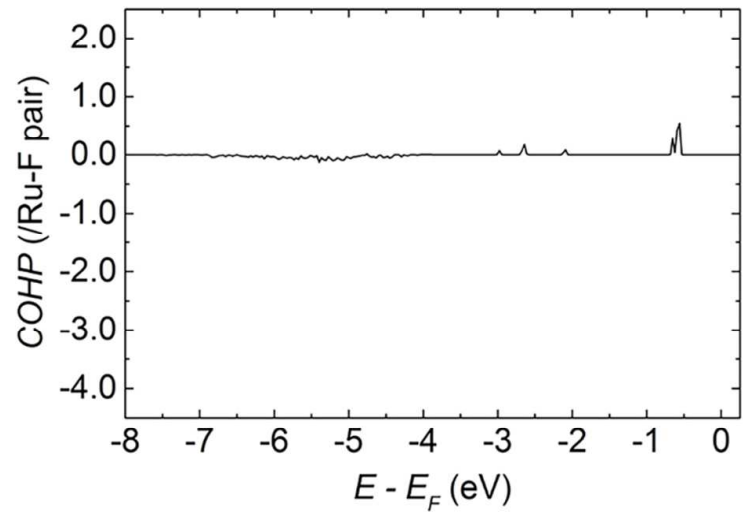

(b)

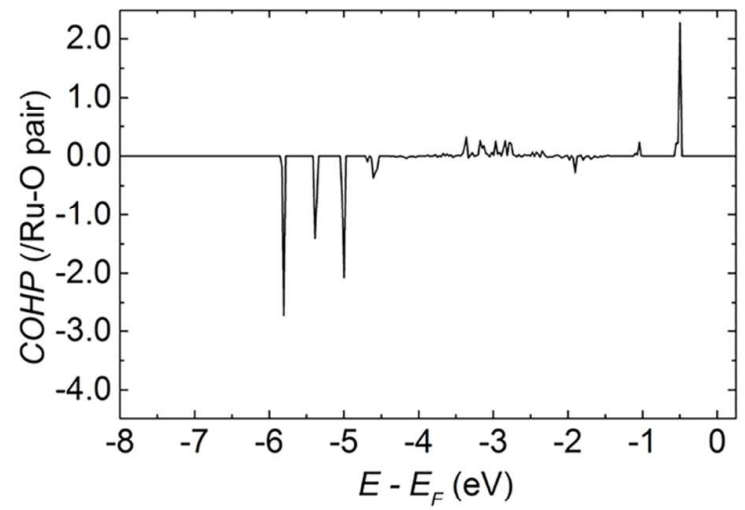

(d)

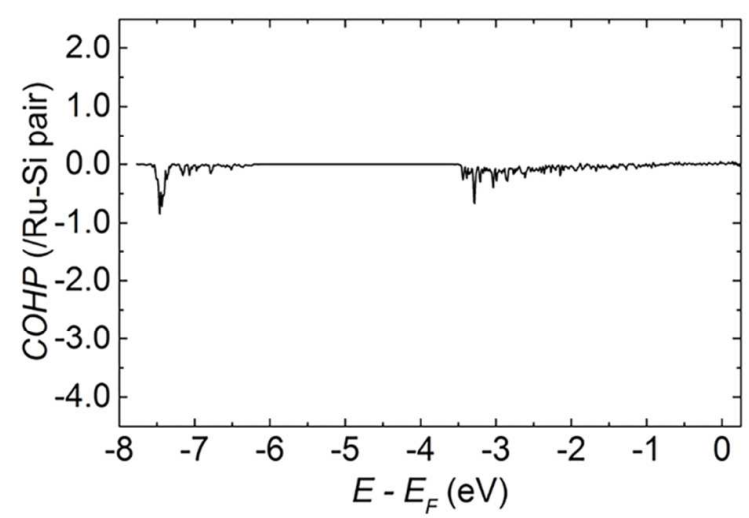

Figure S2. Calculated COHP of (a) $\mathrm{Ru}-\mathrm{N}$ in $\mathrm{Ru}_{1} / \mathrm{Ca}_{3} \mathrm{~N}_{2}$, (b) $\mathrm{Ru}-\mathrm{O}$ in $\mathrm{Ru}_{1} / \mathrm{CaO}$, (c) $\mathrm{Ru}-\mathrm{F}_{\mathrm{ad}}$ in $\mathrm{Ru}_{1} / \mathrm{CaF}_{2}$, and (d) $\mathrm{Ru}-\mathrm{Si}_{\mathrm{ad}}$ in $\mathrm{Ru}_{1} / \mathrm{Ca}_{2} \mathrm{Si}$. 


\section{Ru Migration Pathways}

We calculated the activation energy for a single hopping of a $\mathrm{Ru}$ atom from the most stable adsorption site to the neighboring adsorption site $\left(\Delta E_{\text {mig }}\right)$ in the four supports by using climbing image nudged band elastic (CI-NEB) method ${ }^{2}$ to compare the migration ability of a Ru atom on the four supports. Figure $\mathrm{S} 3$ shows the potential energy profiles of $\mathrm{Ru}$ atom migration on $\mathrm{Ca}_{3} \mathrm{~N}_{2}, \mathrm{CaO}$, $\mathrm{CaF}_{2}$ and $\mathrm{Ca}_{2} \mathrm{Si}$, calculated using VASP. The side views of the surface structures of initial, transition and final states of $\mathrm{Ru}-\mathrm{Ca}_{3} \mathrm{~N}_{2}, \mathrm{CaO}, \mathrm{CaF}_{2}$ and $\mathrm{Ca}_{2} \mathrm{Si}$, during the migration are also shown in the same figures. Calculated $\Delta E_{\text {mig }}$ of $\mathrm{Ru}$ atom on $\mathrm{Ca}_{3} \mathrm{~N}_{2}, \mathrm{CaO}, \mathrm{CaF}_{2}$ and $\mathrm{Ca}_{2} \mathrm{Si}$ are $3.88,1.19,0.05$ and 3.19 $\mathrm{eV}$, respectively. 
(a)

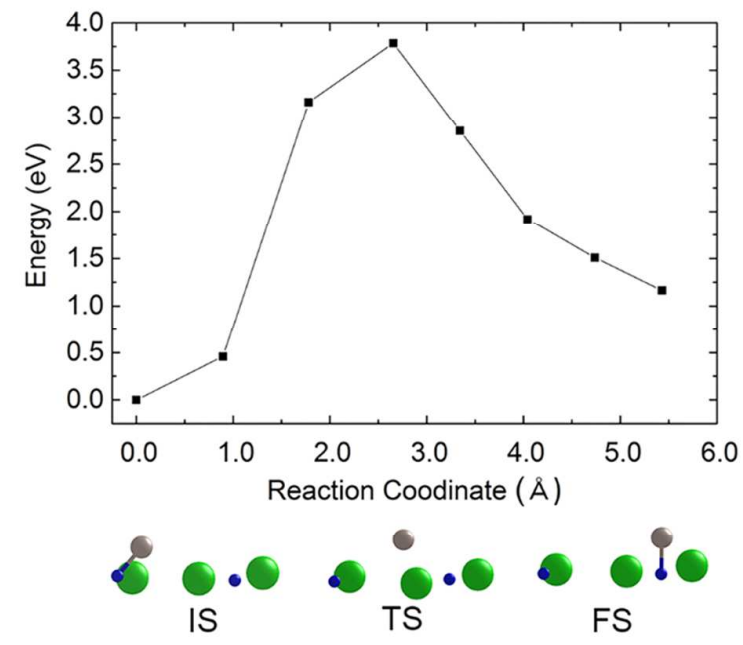

(c)

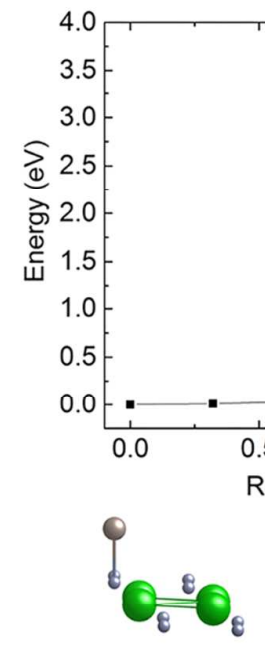

IS
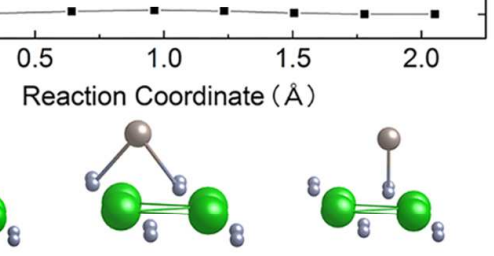

TS

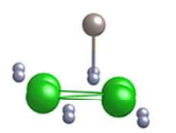

FS (b)
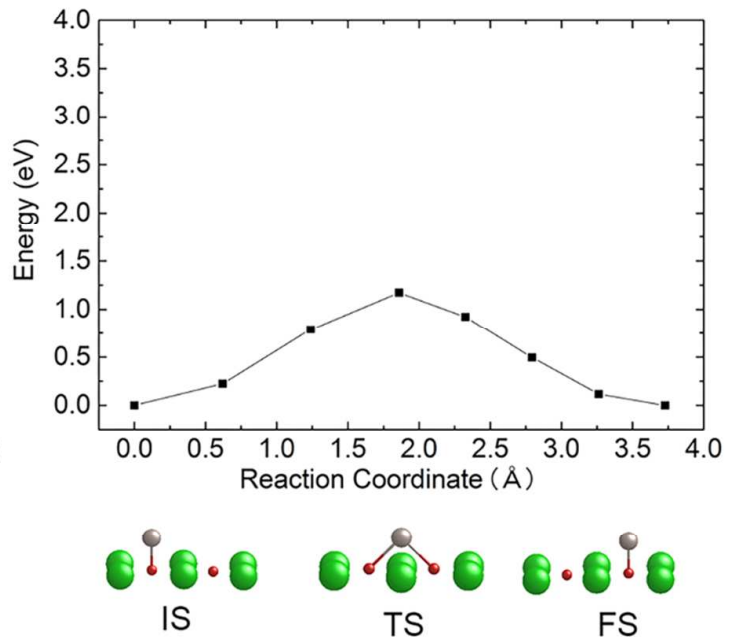

(d)
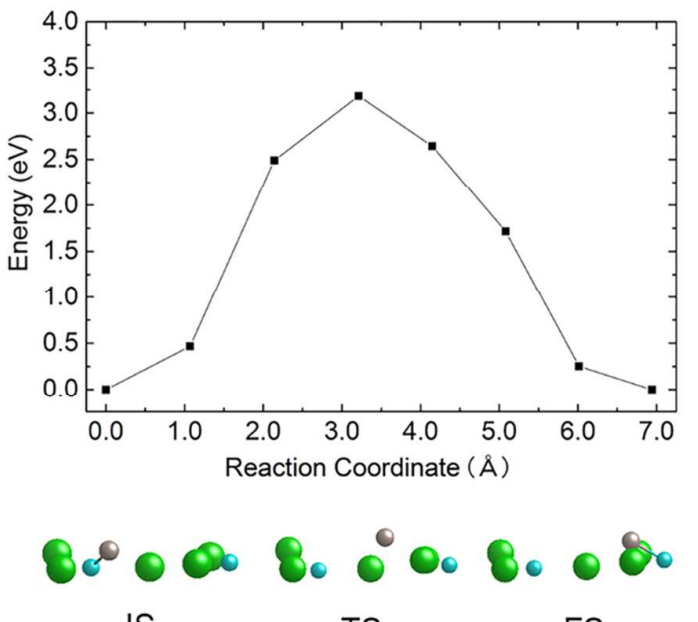

IS

TS

Figure S3. Calculated potential energy profiles of Ru atom migration and the surface structures (side view) of initial, transition and final states for (a) $\mathrm{Ca}_{3} \mathrm{~N}_{2}$, (b) $\mathrm{CaO}$, (c) $\mathrm{CaF}_{2}$, and (d) $\mathrm{Ca}_{2} \mathrm{Si}$.

\section{Transmission Electron Microscope (TEM) and Scanning TEM (STEM) Images}

\section{of Ru Loaded the Supports}

Figure $\mathrm{S} 4$ shows the STEM and TEM images of $\mathrm{Ru}(2 \mathrm{wt} \%) / \mathrm{Ca}_{3} \mathrm{~N}_{2}, \mathrm{CaO}, \mathrm{CaF}_{2}$ and $\mathrm{Ca}_{2} \mathrm{Si}$ powder. 
Round-shaped Ru nanoparticles smaller than $15 \mathrm{~nm}$ were dispersed widely on both $\mathrm{Ca}_{3} \mathrm{~N}_{2}$ and $\mathrm{Ca}_{2} \mathrm{Si}$, which suggests that the Ru nanoparticles are strongly bound to these supports. On the other hand, needle-like $\mathrm{Ru}$ particles $\left(>10 \mathrm{~nm}\right.$ ) were observed on $\mathrm{CaO}$ and $\mathrm{CaF}_{2}$ and these particles make contact with each other to form large Ru aggregates.

(a)

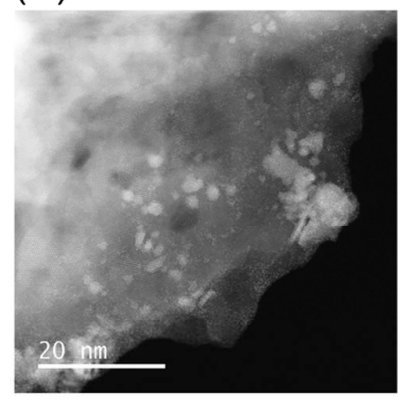

(e)

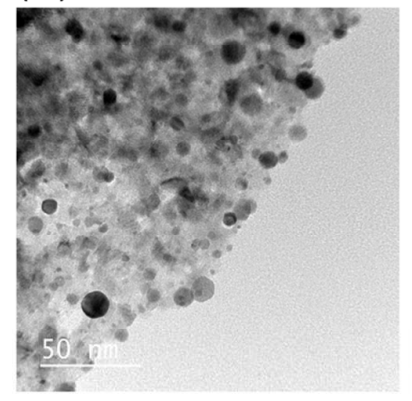

(b)

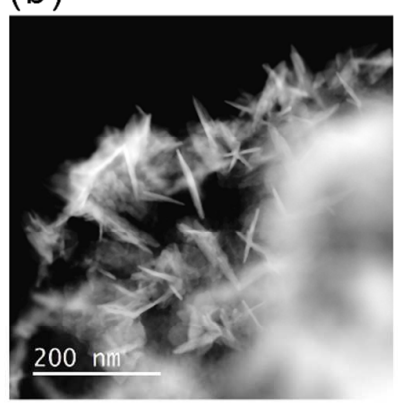

(f)

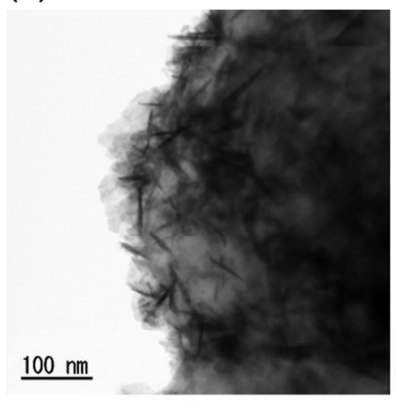

(c)

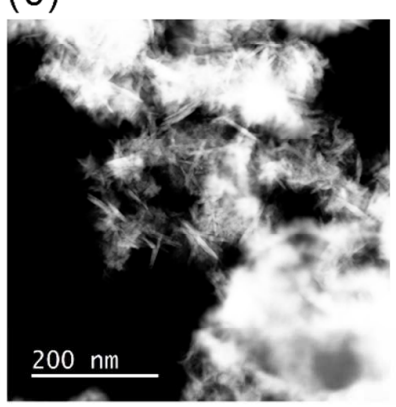

(g)

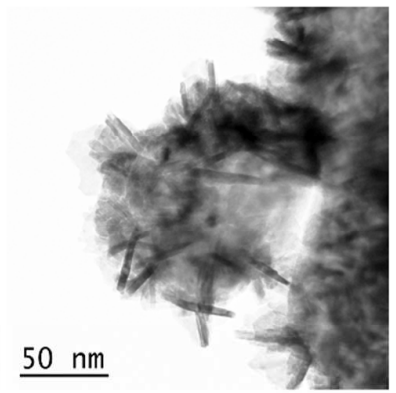

(d)

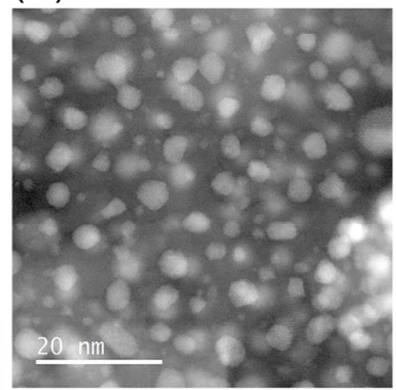

(h)

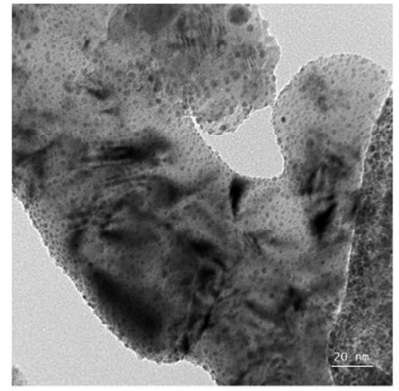

Figure S4. HAADF-STEM images (a-d) and TEM images (e-h) of Ru particles on $\mathrm{Ca}_{3} \mathrm{~N}_{2}$, on $\mathrm{CaO}$, on $\mathrm{CaF}_{2}$ and on $\mathrm{Ca}_{2} \mathrm{Si}$. 


\section{References}

(1) Puchin, V. E.; Puchina, A. V.; Huisinga, M.; Reichling, M. Theoretical Modelling of Steps on the $\mathrm{CaF}_{2}$ (111) Surface. J. Phys. Condens. Matter 2001, 13, 2081-2094.

(2) Henkelman, G.; Uberuaga, B. P.; Jónsson, H. A Climbing Image Nudged Elastic Band Method for Finding Saddle Points and Minimum Energy Paths. J. Chem. Phys. 2000, 113, 9901-9904. 